\title{
Análise epidemiológica da tuberculose em São Luís - MA
}

\author{
Epidemiological analysis of tuberculosis in São Luís - MA \\ Francisca Bruna Arruda Aragão ${ }^{1}$ (D), Ricardo Alexandre Arcêncio² (D), Tânia Gomes Carneiro ${ }^{3}$ (D), \\ Fernanda Carla de Assis Cândido 4 (D), José Henrique da Silva Cunha ${ }^{5}$ (D), Andréa Dias Reis ${ }^{6}$ (D), \\ Regina Célia Fiorati7 (D)
}

\section{RESUMO}

Objetivo: Analisar as ocorrências de Tuberculose registradas em São Luís. Método: Trata-se de um estudo epidemiológico descritivo, com dados obtidos do Sistema de Informação de Agravos de Notificação (SINAN/Tuberculose) de 2014 a 2016. Aplicou-se estatística descritiva com cálculo de frequência absoluta e relativa para variáveis categóricas e medidas de posição e de dispersão para variáveis métricas. Resultados: Foram encontrados 2.306 casos em 378 bairros residenciais. Em 2014, o Distrito mais afetado foi a Vila Cidade Olímpica com 50 casos; em 2015, houve 39 casos e em 2016 ficou com 25 casos. Conclusão: O estudo avança no conhecimento na medida em que apresenta o perfil epidemiológico dos casos de tuberculose em São Luís. Observou-se que a maioria era do sexo masculino, em idade economicamente ativa, da raça/cor parda e com ensino médio completo.

Palavras-Chave: Tuberculose; Epidemiologia; Determinantes Sociais; Avaliação em Saúde; Prevalência.

\section{ABSTRACT}

Objective: Analyzing the occurrence of Tuberculosis registered in São Luís. Method: This is a descriptive epidemiological study, with data obtained from the Notifiable Diseases Information System (SINAN/Tuberculosis) from 2014 to 2016. Descriptive statistics with the calculation of absolute and relative frequency was applied for categorical variables and position and dispersion measures for metric variables. Results: 2,306 cases were found in 378 residential neighborhoods. In 2014, the most affected District was the Vila Cidade Olímpica with 50 cases; in 2015, it had 39 cases, and in 2016 it had 25 cases. Conclusion: The study broadens the knowledge since it presents the epidemiological profile of Tuberculosis cases in São Luís. It was observed that the majority of cases were male, with economically active age, brown ethnic group, and complete secondary education.

Keywords: Tuberculosis; Epidemiology; Social Determinants; Health Evaluation; Prevalence.

1. Enfermeira. Doutoranda em Enfermagem no Programa de Pós-Graduação Interunidades. Escola de Enfermagem de Ribeirão Preto da Universidade de São Paulo (EERP-USP), Ribeirão Preto (SP), Brasil.

2. Enfermeiro. Doutor em Saúde Pública. Docente do Departamento de Enfermagem em Saúde Pública da (EERP-USP), Ribeirão Preto (SP), Brasil.

3. Enfermeira. Doutora em Saúde Pública. Faculdade de Medicina de Ribeirão Preto da Universidade de São Paulo (FMRP-USP), Ribeirão Preto (SP), Brasil.

4. Psicóloga. Mestra em Ciências pelo Programa de Pós-Graduação Enfermagem Psiquiátrica. (EERP-USP), Ribeirão Preto (SP), Brasil.

5. Terapeuta Ocupacional. Mestre em Atenção à Saúde. Programa de Pós-Graduação Enfermagem Psiquiátrica. (EERP-USP), Ribeirão Preto (SP), Brasil.

6. Professora de Educação Física. Doutoranda no Programa de Pós-Graduação em Ciências da Motricidade. Universidade Estadual Paulista "Júlio de Mesquita Filho" (UNESP). Faculdade de Ciências e Tecnologia. Presidente Prudente (SP), Brasil.

7. Terapeuta Ocupacional. Doutora em Ciências. Docente no Curso de Terapia Ocupacional da (FMRP-USP), Ribeirão Preto (SP), Brasil.

$\triangle$ Francisca Bruna Arruda Aragão. Escola de Enfermagem de Ribeirão Preto, Universidade de São Paulo (USP). Rua monte Alegre, no 3900. CEP: 14040-902. Ribeirão Preto (SP), Brasil.

aragao_bruna@usp.br | Recebido em: 17/12/2019 | Aprovado em: 09/07/2020 


\section{INTRODUÇÃO}

A tuberculose (TB) representa um forte desafio para a gestão do cuidado na saúde pública e constitui uma das principais causas de óbitos dentre as doenças transmissíveis. Seus altos índices de prevalência com altos e crescentes níveis de disseminação e morbimortalidade, principalmente em países mais desfavorecidos, representa um dos agentes infecciosos que mais mata, superando o HIV ${ }^{1-3}$.

O Brasil está na $20^{a}$ posição mundial, com uma incidência de 41 casos por 100.000 habitantes e mortalidade de 2,7 óbitos para cada 100.000 habitantes. A Organização Mundial da Saúde (OMS), em 2015, promoveu a estratégia "End TB", com a finalidade de eliminar a TB ( $<1$ caso por 100.000 habitantes), reduzindo em $95 \%$ dos óbitos até $2035^{4}$, incluindo metas claras que, para serem alcançadas, será necessária muita determinação das políticas públicas, com inovação e investimento ${ }^{5}$.

As metas possuem três pilares como base, que são respectivamente: cuidados e prevenção integrados e centrados no paciente; políticas ousadas e sistemas de apoio e suporte aos afetados pela TB; e intensificação da inovação e da pesquisa. Dentre os 30 países com maior incidência de TB, 0 Brasil apresenta menor taxa, inclusive para mortalidade, uma vez que conta com condições técnicas e estrutura para sua erradicação, cobertura universal e acesso ao diagnóstico e tratamento com oferta gratuita pelo Sistema Único de Saúde (SUS) ${ }^{5}$.

Para responder a sinergia entre atores e organizações, estão os serviços de saúde para diagnósticos e tratamentos rápidos e novos métodos para identificação de resistência bacteriana, atrelada às medidas de proteção social e suporte aos pacientes, com impacto em seus contextos ${ }^{5,6}$.

A literatura, de forma marcante, evidencia os determinantes sociais da saúde (que influenciam na demora do diagnóstico, abandono e insucesso do tratamento, multidroga resistência da TB e nos casos de óbito) $)^{1,7,8}$, como fator preponderante na cadeia de progressão da tuberculose, isso circunstancial às precárias condições de domicílio, instabilidade alimentar/ nutricional e inacessibilidade aos serviços de saúde ${ }^{9}$.

Outra questão é a adesão do paciente ao esquema, que é socialmente determinada. Alguns autores ressaltam a importância de coordenação pela Atenção Primária à Saúde para o diagnóstico prévio e pertinente tratamento para redução dos riscos de transmissibilidade ao entorno geográfico do paciente e de sua comunidade ${ }^{5,10}$.

No Maranhão, em 2014, foram notificados 2.237 casos de tuberculose, dentre os quais $82 \%$ foram novos e $11 \%$ retratamentos, com incidência de 26,8 por 100.000 habitantes, ficando em $19^{\circ}$ lugar em comparação aos demais estados e com uma taxa de mortalidade de 2,1/100.000 hab. Em São Luís, houve uma predominância de 4,19/10.000 habitantes, com um índice crescente no gênero feminino, o que denota tratar-se de uma patologia que ainda causa importante problemática social na localidade ${ }^{11}$.

O Plano Estadual de Saúde do Estado do Maranhão 2016-201912 aponta que nos anos de 2010 a 2014 houve uma tendência decrescente dos casos pulmonares bacilíferos, 20,6/100.000 habitantes a 14,8/100.000 habitantes, apresentando algumas oscilações ao longo dos anos no estado e na maioria das regiões de saúde ${ }^{11}$. Contudo, pelos dados do Sistema de Informação de Agravos de Notificação (SINAN/Tuberculose) ${ }^{13}$, percebe-se um aumento do número de casos em São Luís, passando de 661 casos em 2014 para 880 no ano de 2016, demonstrando a importância do desenvolvimento de ações dos gestores do estado do Maranhão para a prevenção à tuberculose nesse referido município ${ }^{11}$.

Nosso estudo utilizou os dados do SINAN ${ }^{13}$, confirmando a crescente incidência de casos novos de TB, além de analisar os fatores sociodemográficos e variáveis de caracterização dos pacientes de tuberculose. Destacamos que as taxas de incidência estão diretamente relacionadas à cobertura das ações de deteç̧ão de casos novos para TB nas Unidades Básicas de Saúde, eficácia da notificação em tempo oportuno. A falta de continuidade dessas ações no nível municipal impacta diretamente nesse indicador. Pelo exposto, o estudo tem o objetivo de analisar as ocorrências de Tuberculose registradas em São Luís.

\section{MÉTODO}

\section{Desenho do estudo}

Trata-se de um estudo epidemiológico descritivo ${ }^{14}$. 


\section{Contexto (setting)}

O município de São Luís, região metropolitana com 1.101.884 habitantes, uma área de $582,974 \mathrm{~km}^{2}$, está localizado ao norte do Estado do Maranhão, ao derredor dos municípios de Raposa, Paço do Lumiar e São José de Ribamar ${ }^{15}$.

As ocorrências registradas dos casos de Tuberculose em São Luís, de 2014 a 2016, foram notificados nos sete distritos sanitários de saúde, que são constituídos por 211 bairros residenciais, sendo: Distrito Centro com 14 bairros; Itaqui-Bacanga com 32; Coroadinho com 29; Cohab com 35; Bequimão com 31; Tirirical com 42 e Vila Esperança com 28 bairros $^{13}$.

\section{População do Estudo}

A população foi constituída pelos indivíduos diagnosticados com tuberculose, residentes em sete distritos sanitários, os pacientes tinham ficha de registro no SINAN, contendo as seguintes variáveis: faixa etária, sexo, raça, escolaridade e residência, frequência por tipo de entrada, forma clínica e caso novo por baciloscopia de escarro.

Os casos novos foram calculados, conforme a base de cálculo amostral do SINAN ${ }^{13}$, que corresponde a identificar primeiro o indicador que deseja avaliar com descrição do período, seguido do cálculo de número de casos novos no município (SINAN), dividido pelo total de casos do mesmo município de acordo com o Instituto Brasileiro de Geografia Estatística e o Tribunal de Contas da União (IBGE e TCU), multiplicado por 100.000 , dados correspondentes a um ano e o cálculo conforme a adição do período e coeficiente desejado.

Os casos novos foram caracterizados como qualquer caso que nunca utilizou a medicação antituberculosa, ou a utilizou por menos de 30 dias. O SINAN ${ }^{13}$ verifica se o paciente e seus familiares não tiveram tratamento prévio para tuberculose por 30 dias ou mais.

\section{Tamanho do estudo}

O tamanho da amostra foi determinado a partir dos registros de 2.306 pessoas das unidades sanitárias de 7 distritos que cobrem um total de 211 bairros residenciais.

\section{Fonte de Dados}

Foram utilizados como dados as notificações em São Luís, pelo Sistema de Informação de Agravos de Notificação (SINAN/Tuberculose) demonstrados na forma de planilha eletrônica Microsoft Office Excel $2008 \AA$, agrupados em sete distritos sanitários.

\section{Variáveis}

Os pacientes apresentam sintomas clínicos gerais, buscando serviços como a atenção primária e, apesar de existirem condições técnicas e estrutura para sua erradicação e fácil acesso ao diagnóstico e tratamento com oferta gratuita pelo Sistema Único de Saúde (SUS), um agravante neste contexto é a descontinuidade do tratamento.

Os desfechos foram divididos em duas categorias: sociodemográficas (sexo, raça/cor e escolaridade) e epidemiológicas (casos novos por: faixa-etária, distrito de residência, forma clínica e baciloscopia de escarro).

\section{Variáveis quantitativas}

As variáveis quantitativas foram transformadas em percentuais a partir da respectiva quantidade de pessoas identificadas no relatório do SINAN/ Tuberculose.

\section{Teste Rápido Molecular}

O Teste Rápido Molecular para Tuberculose (RTR-TB) teve sua implementação a partir de julho de 2014, com ampla expansão em todas as cidades brasileiras e São Luís teve seu equipamento implantado logo no primeiro ano de implantação da RTR-TB ${ }^{16}$.

\section{Plano de Análise}

Os dados foram analisados no programa Excel (Microsoft Office $\Re$ 2007), na construção das Tabelas 1 e 2. Aplicou-se estatística descritiva, com 
cálculo das medidas de posição (média) e dispersão (desvio-padrão), para as variáveis qualitativas foram computadas medidas de frequência absoluta e relativa.

\section{Aspectos Éticos da Pesquisa}

Todos os procedimentos que envolvem seres humanos foram preservados, conforme preconizado nas "Diretrizes e Normas Regulamentadoras de Pesquisa Envolvendo Seres Humanos" do Conselho Nacional de Saúde n466/2012. A pesquisa foi apreciada e aprovada pelo Comitê de Ética em Pesquisa da Escola de Enfermagem de Ribeirão Preto da Universidade de São Paulo - USP com parecer de número 3.411.398.

\section{RESULTADOS}

A Tabela 1 apresenta as características sociodemográficas de casos novos de tuberculose entre 2014 a 2016, sendo $67,22 \%$ pacientes do sexo masculino e a maioria de raça/cor parda $(n=1747$; $75,76 \%)$ e a menor quantidade indígena $(n=11$; $0,48 \%)$.

Em relação à escolaridade, a maior proporção tinha o ensino médio completo $(n=620$; $26,89 \%$ ), seguido dos pacientes que tinham entre $5^{a}$ e $8^{a}$ série incompleta $(n=428 ; 18,56 \%)$ e aqueles com $1^{\text {a }}$ a $4^{a}$ série incompleta $(n=264$; $11,45 \%)$. Dessa forma, observa-se que a maior prevalência de casos novos de TB foram das pessoas que apresentavam ensino médio completo. Os bairros de maior incidência entre os anos pesquisados são bairros populares, que englobam áreas de população com menor escolaridade, denotando que dentre os indicadores mais importantes para mensurar o nível socioeconômico associado à saúde da população está o nível de instrução (Tabela 1).

Foram identificados pacientes acometidos por Tuberculose em sua maioria com idade média entre 20 e 34 anos com um quantitativo de 935 pessoas que corresponde ao percentual de 40,55\%. Em cada distrito de residência, foram identificados a maioria dos casos nos bairros Cohab $(n=362 ; 15,70 \%)$ e Tirirical $(n=362 ; 15,70 \%)$ com o mesmo quantitativo registrado e ficaram atrás somente para o bairro do Coroadinho ( $n=353 ; 15,31 \%)$ (Tabela 2$)$.

\section{Tabela 1}

Caracterização Sociodemográfica de casos novos de tuberculose em São Luís-MA, Brasil, 2014 a 2016 $(n=2.306)$

\begin{tabular}{|c|c|c|}
\hline Sexo & $\mathbf{n}$ & $\%$ \\
\hline Masculino & 1.550 & 67,22 \\
\hline Feminino & 756 & 32,78 \\
\hline Raça/Cor & $\mathbf{n}$ & $\%$ \\
\hline Ignorado/Branco & 17 & 0,74 \\
\hline Branca & 258 & 11,19 \\
\hline Negra & 261 & 11,32 \\
\hline Amarela & 12 & 0,52 \\
\hline Parda & 1.747 & 75,76 \\
\hline Indígena & 11 & 0,48 \\
\hline Escolaridade & $\mathbf{n}$ & $\%$ \\
\hline Ignorado/Branco & 115 & 4,99 \\
\hline Analfabeto & 70 & 3,04 \\
\hline $1^{a}$ a $4^{a}$ série Incompleta & 264 & 11,45 \\
\hline $4^{a}$ série Completa & 155 & 6,72 \\
\hline $5^{a}$ a $8^{a}$ série Incompleta & 428 & 18,56 \\
\hline Ensino Fundamental Completo & 180 & 7,81 \\
\hline Ensino Médio Incompleto & 250 & 10,84 \\
\hline Ensino Médio Completo & 620 & 26,89 \\
\hline Ensino Superior Incompleto & 72 & 3,12 \\
\hline Ensino Superior Completo & 111 & 4,81 \\
\hline Ignorado & 41 & 1,78 \\
\hline
\end{tabular}

*Dados expressos por frequência absoluta e relativa.

Dentre os casos notificados dos pacientes com TB, os casos novos por baciloscopia de escarro na sua maioria com exame baciloscópico de escarro positivo com um quantitativo de 1.048 pessoas, referente a um percentual de $45,45 \%$, pois estes são os casos que transmitem mais a doença e são especialmente relevantes do ponto de vista da saúde pública, sendo os casos negativos registrados somente um quantitativo de 485 casos, que em percentuais fica em 21,03\% (Tabela 2). 


\section{Tabela 2}

Análise de casos novos de tuberculose em São Luís-MA, Brasil, 2014 a $2016(n=2.306)$

\begin{tabular}{|c|c|c|}
\hline Caso Novo por & $\mathbf{n}$ & $\%$ \\
\hline Faixa-etária & Média & Desvio padrão \\
\hline$<1$ Ano & 6,75 & 4,21 \\
\hline $1-4$ & 2,00 & 2,12 \\
\hline $5-9$ & 0,75 & 0,83 \\
\hline $10-14$ & 7,00 & 4,95 \\
\hline $15-19$ & 37,00 & 4,95 \\
\hline $20-34$ & 233,75 & 15,85 \\
\hline $35-49$ & 151,5 & 16,52 \\
\hline $50-64$ & 89,75 & 14,62 \\
\hline $65-79$ & 38,25 & 5,36 \\
\hline$>80$ & 9,75 & 4,26 \\
\hline Distrito de Residência & Média & Desvio padrão \\
\hline Tirirical & 90,50 & 12,84 \\
\hline Centro & 63,25 & 10,62 \\
\hline Coroadinho & 88,25 & 9,28 \\
\hline Vila Esperança & 83,75 & 12,74 \\
\hline Bequimão & 71,50 & 3,20 \\
\hline Cohab & 90,50 & 12,05 \\
\hline Itaqui-Bacanga & 67,00 & 7,97 \\
\hline Outros & 21,75 & 16,56 \\
\hline Forma Clínica & Média & Desvio padrão \\
\hline Pulmonar & 494,00 & 41,70 \\
\hline Extrapulmonar & 79,00 & 7,28 \\
\hline \multirow[t]{2}{*}{ Pulmonar + Extrapulmonar } & 3,50 & 1,50 \\
\hline & Média & Desvio padrão \\
\hline Ignorado/Branco & 12,50 & 16,86 \\
\hline Positivo & 262,00 & 74,81 \\
\hline Negativo & 121,25 & 37,57 \\
\hline Não Realizado & 180,75 & 67,81 \\
\hline
\end{tabular}

*Dados expressos por média e desvio-padrão.

\section{DISCUSSÃO}

Foi encontrado em cada distrito de residência, a maioria dos casos nos bairros Cohab $(n=362$;
$15,70 \%)$ e Tirirical $(n=362 ; 15,70 \%)$, com o mesmo quantitativo registrado. Não obstante seja possível evitar a TB, tendo esta um diagnóstico relativamente simples, curável e que hoje conta com um tratamento universal e disponível pela rede pública de saúde, o que deveria beneficiar a população, ainda existe um forte desafio, pois em 2016 foi estimado que 10,4 milhões de pessoas adoeceram e 1,7 milhões de pessoas foram a óbito no mundo em consequência desta doença (incluindo $400 \mathrm{mil}$ coinfectadas com TB/HIV) 6 .

A faixa etária de maior risco identificada neste estudo foi entre 20 e 34 anos, que representa um percentual de $40,55 \%$. Historicamente, a tuberculose pulmonar em adultos tem tido maior enfoque por se tratar de doença mais infecciosa, pois transmite a doença por meio de partículas infecciosas de aerossol, que são tipicamente produzidas quando este tipo de paciente tosse ${ }^{17}$.

A TB atinge os indivíduos economicamente ativos, principalmente homens que estão na faixa etária produtiva (registrado $60,22 \%$ neste estudo), abandonando o tratamento com maior facilidade que os de sexo feminino, apresentando diferenças estatísticas consideráveis ${ }^{7}$. A literatura aponta que o homem tem maior tendência à descontinuidade no tratamento da TB, e é pertinente considerar que estes estão em idade ativa e assumem a principal responsabilidade pelo sustento financeiro familiar, preocupando-se muito menos com sua própria saúde ${ }^{18,19}$.

Outrossim, pessoas com menor renda e escolaridade (este estudo apontou 26,89\% com Ensino Médio completo e $18,56 \%$ até a $8^{a}$ série incompleta) são mais fragilizadas ao agravamento da doença, com falhas terapêuticas e a não adesão ao tratamento, pois geralmente se relacionam também à falta de conhecimento sobre a doença, custos terapêuticos para si e sua família ${ }^{18}$, um forte indicativo está vinculado à baixa escolaridade pela própria condição social das pessoas acometidas ${ }^{1}$.

Quanto ao Distrito de Residência, de acordo com o IDSE (Índice de Desigualdade Socioespacial de São Luís) por bairros, Coroadinho (15,31\%) e São Cristóvão (Tirirical com 15,70\%) elencam os bairros mais desfavorecidos conjuntamente com Vila Mauro Fecury, Campus/UFMA, Sá Viana, Olho d'Água, Vila Embratel, Vila Bacanga, Cidade Operária e Pindorama e todos estão abaixo do IDSE 
como um todo juntamente com o bairro mais segregado que é a Vila Nova ${ }^{19}$.

Convém salientar que a forma clínica pulmonar, conforme identificado neste estudo, representa a maioria dos casos detectados e aproximadamente $85,69 \%$ dos casos de TB são pulmonares, e os pacientes apresentam sintomas clínicos gerais, fazendo com que busquem serviços como a atenção primária, à saúde materno-infantil (no caso das crianças) ou clínicas gerais ${ }^{6}$, porém ainda concentra as maiores taxas de abandono do tratamento ${ }^{18}$.

Durante o tratamento, os pacientes não precisam ser segregados da convivência familiar e da comunidade, uma vez que, após seu início, não deve haver interrupção, exceto se uma revisão clínica for feita com rigor e indicar que seja alterado o diagnóstico ${ }^{20}$.

Os pacientes bacilíferos que não continuam o tratamento não conseguem interromper a cadeia epidemiológica da $T B$, e continuam transmitindo o bacilo, infectando outras pessoas, podendo ainda desenvolver formas mais potenciais e resistentes da infecção ${ }^{18}$. Por esta razão, não priorizar este tipo de exame, independente das inovações tecnológicas introduzidas para identificação diagnóstica, a qualidade da amostra de escarro é prioritária, pois qualquer resultado laboratorial depende da qualidade da amostra coletada, apesar de que sua não priorização pode ser em decorrência de falhas na sua operacionalização, na disponibilidade de insumos até a logística para o processamento do exame ${ }^{8,21}$.

Diversos achados sobre a epidemiologia da doença apontam os principais segmentos sociais atingidos pela TB, que são: pessoas com baixa renda, migrantes e pessoas coinfectadas por tuberculose e HIV, relacionando a pobreza, exclusão social e miséria, desigualdade social (barreiras sociais, econômicas, demográficas e cultural; a escolaridade; problemas inerentes ao medicamento) e o êxito do tratamento depende da adesão do paciente, além dos fatores anteriormente elencados, a falta de informação da família, uso de álcool e drogas ilícitas ${ }^{2,10,19}$.

As limitações deste estudo foram a falta de acesso aos dados de coinfecção por HIV e informações socioeconômicas nos registros do SINAN nos anos de 2014 a 2016, na ficha critério laboratorial e critério clínico-epidemiológico. Entretanto, os resultados apontados neste estudo poderão servir como orientação para gestores e profissionais de saúde, em relação aos locais prioritários que necessitam de maior atenção na luta contra a TB, por muitas vezes evitando óbitos. Desta forma, este trabalho aponta aspectos relevantes a serem considerados sobre esta realidade, tanto sob o ponto de vista clínico, quanto ao ambiente na efetividade de estratégias para reduzir as ocorrências da doença.

\section{CONCLUSÃO}

Verificou-se, neste estudo, que o perfil epidemiológico de indivíduos acometidos por tuberculose na cidade de São Luís do Maranhão foi de indivíduos adultos do sexo masculino, em idade economicamente ativa, da raça/cor parda, com ensino médio completo, com forma clínica pulmonar, em condições socioeconômicas desfavoráveis. Em cada distrito de residência, identificou-se a maioria dos casos nos bairros Cohab e Tirirical.

Assim sendo, no município investigado, é necessária a adoção de estratégias que visem melhorar o controle da tuberculose e reduzir sua disseminação e, consequentemente, o número de óbitos ocasionados pela doença.

\section{CONFLITO DE INTERESSE} interesses.

Os autores declaram não haver conflito de

\section{AGRADECIMENTOS}

À Coordenação de Aperfeiçoamento de Pessoal de Nível Superior (CAPES).

\section{CONTRIBUIÇÃO DOS AUTORES}

Aragão, FBA e Arcêncio, RA contribuíram na aquisição, análise, interpretação de dados do estudo; Carneiro, TG, Cândido, FCA, Cunha, JHS e Reis, $A D$ contribuíram na análise e interpretação de dados do estudo; e Fiorati, RC contribui na aprovação da versão final do estudo a ser publicado. 


\section{REFERÊNCIAS}

1. Touso MM, Popolin MP, Crispim JDA, Freitas IMD, Rodrigues LBB, Yamamura M, et al. Estigma social e as famílias de doentes com tuberculose: um estudo a partir das análises de agrupamento e de correspondência múltipla. Ciên Saúde Colet. [Internet]. 2014 [citado em 22 jul 2019]; 19(11):4577-4585. Disponível em: http:// dx.doi.org/10.1590/1413-812320141911.46062013.

2. Ceccon RF, Maffacciolli R, Burile A, Meneghel SN, Oliveira DLLC, Gerhardt TE. Mortalidade por tuberculose nas capitais brasileiras, 2008-2010. Epidemiol Serv Saude. [Internet]. 2017 [citado em 25 jul 2019]; 26(2):349358. Disponível em: https://doi.org/10.5123/S167949742017000200012.

3. Ministério da Saúde (BR). Secretaria de Vigilância em Saúde. Departamento de Vigilância epidemiológica. Implantação do plano nacional pelo fim da tuberculose como problema de saúde pública no Brasil: primeiros passos rumo ao alcance das metas. Boletim Epidemiológico. [Internet]. 2018 [citado em 25 jul 2019]; 49(11): 1-18. Disponível em: http://portalarquivos2.saude.gov.br/ images/pdf/2018/marco/26/2018-009.pdf.

4. Fiorati RC, Cândido FCA, Souza LB, Popolin MP, Ramos ACV, Arcêncio RA. Desigualdades sociais e os desafios à estratégia de eliminação da tuberculose no Brasil. Vittalle Revista de Ciências da Saúde. [Internet]. 2018 [citado em 22 jul 2019]; 30(2): 59-72. Disponível em: https://doi.org/10.14295/vittalle.v30i2.7502.

5. Barreira D. Os desafios para a eliminação da tuberculose no Brasil. Epidemiol Serv Saude. [Internet]. 2018 [citado em 25 jul 2019]; 27(1):e00100009. Disponível em: http://www.scielo.br/pdf/ress/v27n1/2237-9622ress-27-01-e00100009.pdf.

6. Arroyo LH, Yamamura M, Protti-Zanatta ST, Fusco APB, Palha PF, Ramos $A C V$, et al. Identificação de áreas de risco para a transmissão da tuberculose no município de São Carlos, São Paulo, 2008 a 2013. Epidemiol Serv Saude. [Internet]. 2017 [citado em 22 jul 2019]; 26(3):525-534. Disponível em: https://dx.doi. org/10.5123/s1679-49742017000300010.

7. Moraes MFV, Corrêa RGCF, Coutinho NPS, Caldas AJM, Silva TC, Santos KCB, et al. Perfil epidemiológico de casos de tuberculose em um município prioritário no Estado do Maranhão. Rev Pesq Saúde. [Internet]. 2017 [citado em 22 jul 2019]; 18(3): 147-150. Disponível em: http://www.periodicoseletronicos.ufma.br/index. $\mathrm{php/revistahuufma/article/view/10149}$

8. Sicsú NA, Salem JI, Fujimoto LBM, Gonzales RIC, Cardoso MSL, Palha PF. Intervenção educativa para a coleta de escarro da tuberculose: um estudo quase experimental. Rev Latino-Am Enfermagem. [Internet]. 2016 [citado em 22 jul 2019]; 24:e2703. Disponível em: http://www. scielo.br/pdf/rlae/v24/pt_0104-1169-rlae-24-02703.pdf.

9. Andrade KVF, Nery JS, Araújo GS, Barreto ML, Pereira SM. Associação entre desfecho do tratamento, características sociodemográficas e benefícios sociais recebidos por indivíduos com tuberculose em Salvador, Bahia, 2014-2016.
Epidemiol Serv Saude. [Internet]. 2019 [citado em 22 jul 2019]; 28(2):e2018220. Disponível em: http://dx. doi.org/10.5123/s1679-49742019000200004.

10. Souza ACS, Silva MLSJ, Miranda LN. Dificuldades na adesão do plano de tratamento pelo paciente com Tuberculose. Ciências Biológicas e de Saúde Unit. [Internet]. 2018 [citado em 22 jul 2019]; 4(2):297312. Disponível em: https://periodicos.set.edu.br/ index.php/fitsbiosaude/article/view/4560.

11. Secretaria de Saúde do Estado do Maranhão. Plano Estadual de Saúde 2016-2019. Técnicos da Secretaria de Estado da Saúde - SES [Internet]. 2016 [citado em 22 jul 2019]. Disponível em: http://www.transparencia.ma.gov.br/prestacao-de-contas/2016/1.40.01\%20 \%20Plano\%20de\%20sa\%C3\%BAde $\% 20$ e\%20relat\%C3\%B3rio\%20de\%20gest\%C3\%A3o.pdf.

12. Maranhão. Secretaria de Saúde. Plano estadual de saúde: PES 2016-2019. São Luís, 2016. Disponível em: <https://www.conass.org.br/pdf/planos-estaduais-de-saude/MA_Plano\%20de\%20saude\%202016-2019. pdf>. Acesso em: 01 jul. 2020.

13. Ministério da Saúde (BR). Ministério da Saúde/SVS - Sistema de Informação de Agravos de Notificação Sinan Net. [Internet]. 2015 [citado em 22 jul 2019]. Disponível em: http://tabnet.datasus.gov.br/cgi/tabcgi. exe?sinannet/cnv/tubercma.def.

14. Rothman KJ. Epidemiolgy: an introduction. 2th ed. New York: Oxford University Press; 2012.

15. Instituto Brasileiro de Geografia e Estatística (IBGE). Malha municipal do Estado do Maranhão. Divisão PolíticoAdministrativa Brasileira - DPA. Estrutura político-administrativa vigente em 30/04/2018. [Internet]. 2018 [citado em 22 jul 2019]. Disponível em: http://www.ibge. gov.br/home/geociencias/areaterritorial/resolucao.shtm.

16. Ministério da Saúde (BR). Ministério da Saúde. Secretaria de Vigilância em Saúde. Departamento de Vigilância das Doenças Transmissíveis. Rede de teste rápido para tuberculose no Brasil: primeiro ano da implantação. Brasília: Ministério da Saúde; 2015.

17. Velizarova S, Gabrovska N, Spasova A, Marais B, Harausz EP, Tiberi S, et al. Tratamento e desfechos da tuberculose grave em crianças na unidade de terapia intensiva pediátrica: é possível identificar as melhores práticas? J Bras Pneumol. [Internet]. 2019 [citado em 22 jul 2019]; 45(2): e20190043. Disponível em: http://www.scielo.br/scielo.php?script=sci_arttext\&pid $=$ S180637132019000200100\&lng=en\&nr$\mathrm{m}=$ iso\&tlng $=\mathrm{pt}$.

18. Durans JJF, Sá EM, Pereira LFB, Soares DL, Oliveira PS, Aquino DMC, et al. Perfil clínico e sociodemográfico de pacientes que abandonaram o tratamento de tuberculose no município de São Luís - MA. Rev Pesq Saúde. [Internet]. 2013 [citado em 22 jul 2019]; 14(3): 175-178.

19. Yamamura M, Neto MS, Neto FC, Arroyo LH, Ramos ACV, Queiroz AAR, et al. Areas with evidence of equity and their progress on mortality from tuberculosis in an endemic municipality of southeast Brazil. Infect Dis Poverty. [Internet]. 2017 [citado em 22 jul 2019]; 
6(1):134. Disponível em: https://www.ncbi.nlm.nih. gov/pubmed/29020981.

20. Araújo SRL, Pereira ISSD, Brito NOS, Fonseca PCB. Perfil epidemiológico da tuberculose pulmonar na cidade de Natal - RN. J Infect Control. [Internet]. 2015 [citado em 22 jul 2019]; 4(1): 16-19. Disponível em: http:// jic-abih.com.br/index.php/jic/article/view/77.
21. Ponce $M A Z$, Wysocki $A D$, Scatolin BE, Andrade RLP, Arakawa $T$, Netto AR, et al. Diagnóstico da tuberculose: desempenho do primeiro serviço de saúde procurado em São José do Rio Preto, São Paulo, Brasil. Cad Saúde Pública. [Internet]. 2013 [citado em 22 jul 2019]; 29(5):945-954. Disponível em: http://dx.doi. org/10.1590/S0102-311X2013000500012. 